\title{
Early decrease in postoperative serum albumin predicts severe complications in patients with colorectal cancer after curative laparoscopic surgery
}

\author{
Yong Wang ${ }^{\dagger}$, Honggang Wang ${ }^{\dagger}$, Jianguo Jiang ${ }^{\dagger}$, Xiaofei Cao ${ }^{*}$ and Qinghong Liu*
}

\begin{abstract}
Background: Postoperative severe complications are always associated with prolonged hospital stays, increased economic burdens, and poor prognoses in patients with colorectal cancer (CRC). This present study aimed to investigate potential risk factors including serum albumin (Alb) for severe complications in CRC patients.
\end{abstract}

Methods: Eligible patients with primary CRC undergoing elective laparoscopic colectomy from July 2015 to July 2017 were included. Postoperative severe complications were defined as grade III and IV according to the Clavien-Dindo classification. $\triangle \mathrm{Alb}$ was defined as (preoperative Alb - nadir Alb within POD2)/preoperative Alb $\times 100 \%$. The baseline characteristics, intraoperative data, and laboratory data were obtained from the database for the analysis. Univariate and multivariate logistic regression analyses were utilized for the assessment of the association between risk factors and postoperative severe complications. The predictive value of $\triangle \mathrm{Alb}$ for postoperative severe complications was evaluated by receiver operating characteristic (ROC) curve analysis.

Results: A total of 193 patients were finally included in the analysis data set, of which 38 (19.7\%) patients had postoperative severe complications. In the final multivariate logistic regression analysis, $\triangle \mathrm{Alb}$ was the only independent factor associated with postoperative severe complications (OR 1.66,95\% Cl 1.18-2.33, $p=0.003)$. The area under the curve (AUC) of $\triangle$ Alb was 0.916 , with the sensitivity and specificity of 0.842 and $0.858(p<0.001)$.

Conclusions: The $\triangle \mathrm{Alb}$ was an independent risk factor for severe complications in CRC patients after curative laparoscopic surgery.

Keywords: Colorectal cancer, Postoperative complications, Predictor, Albumin

\section{Background}

Colorectal cancer (CRC) has been widely accepted as the third most common malignant neoplasm worldwide with an increasing incidence in recent years [1]. Surgical resection remains the cornerstone curative treatment for $\mathrm{CRC}$, and laparoscopic colorectal surgery is developing constantly in the recent decades. Despite the great improvements in surgical procedures, perioperative managements, and multidisciplinary therapies, postoperative

\footnotetext{
*Correspondence: 2856029308@qq.com; drliuqinghong@126.com

†Yong Wang, Honggang Wang and Jianguo Jiang contributed equally to this work.

Department of General Surgery, Taizhou People's Hospital, Taizhou Clinical Medical College of Nanjing Medical University, Medical School of Nantong University, No.366 Taihu Road, Taizhou 225300, Jiangsu, China
}

severe complications persist to some extent [2]. As illustrated by the previous data, the incidence of postoperative severe complication can reach as high as approximately $25 \%$ [3]. The severe complications are always associated with prolonged hospital stays, increased economic burdens, and poor prognoses [4]. Therefore, to investigate potential factors for postoperative severe complications can help to stratify complication risks and improve clinical decision-making.

After surgery, circulating acute phase proteins, such as interleukin-6 (IL-6) and C-reactive protein (CRP), usually increase because of the surgical stress and proinflammatory cytokines $[5,6]$. Previous data has widely considered these two proteins as potential predictors for

(C) The Author(s). 2018 Open Access This article is distributed under the terms of the Creative Commons Attribution 4.0 International License (http://creativecommons.org/licenses/by/4.0/), which permits unrestricted use, distribution, and 
postoperative complications after elective colorectal surgery [7]. Albumin (Alb), as a negative acute phase protein and nutritional marker, decreases immediately after operation in response to surgical stress. Previous literature has suggested the prognostic role of preoperative hypoalbuminemia in patients undergoing colorectal surgery [8]. However, few studies have focused on the effect of the change of serum Alb on postoperative complications. This present study aimed to investigate potential risk factors including proinflammatory cytokines and nutritional markers for severe complications in CRC patients.

\section{Methods}

\section{Patients}

This retrospective study protocol was approved by the Medical Institutional Ethics Committee of Jiangsu province. Eligible patients with primary CRC undergoing elective laparoscopic colectomy at the Department of General surgery, Taizhou People's Hospital from July 2015 to July 2017 were included. The inclusion criteria were as follows: (1) adult patients aged over 18, (2) first pathologically diagnosed with primary CRC supported by operative and pathological results, and (3) patients who underwent a curative laparoscopic resection of primary tumors for the first time. The exclusion criteria were as follows: (1) with tumor metastasis found either pre-operatively or intra-operatively, (2) with emergency operation due to complications (bowel obstruction, perforation, etc.), (3) with neo-adjuvant treatment, (4) accompanied by other malignancies; (5) and with laparotomy or laparoscopic conversion to laparotomy.

\section{Study design}

The surgical procedures, including the extent of both colectomy and lymph node dissection, were conducted according to the Colorectal Cancer Treatment Guidelines [9]. All the enrolled patients received the same perioperative managements. The diet was not resumed until the patients passed flatus. No patients died within postoperative day (POD) 30 in this present study.

The baseline characteristics (age, gender, etc.), intraoperative data (duration of operation, intraoperative blood transfusion, etc.), and laboratory data (CRP, Alb, etc.) were obtained from the database for the analysis. The pathological classifications were evaluated following the guidance of the 7th edition of American Joint Committee on Cancer (AJCC) TNM Classification.

\section{Definitions and outcomes}

The primary outcome was the occurrence of postoperative complications within postoperative 30 days [10]. Postoperative severe complications were defined as grade III and IV according to the Clavien-Dindo classification [11].
Enrolled patients were initially grouped according to the presence of severe complications.

As reported by previous studies [12], the relative change of the serum Alb ( $\Delta \mathrm{Alb})$ was defined as (preoperative Alb-nadir Alb within POD2)/preoperative Alb $\times 100 \%$. The relative changes of the hemoglobin $(\mathrm{Hb})$ and hematocrit (Hct) were with the same definitions. The median $\Delta$ Alb level was accepted as the cutoff value for the discrimination of high versus low value [7].

\section{Statistical analysis}

Data were analyzed by the SPSS 23.0 (SPSS, Inc., IA, USA) and GraphPad Prism 5.0 (GraphPad Inc., CA, USA), and a $p<0.05$ was considered statistically significant. Before the study, we performed a sample size estimation of 150 patients. According to our clinical experience and previously published reports [7], the estimated incidence of postoperative severe complications was used as a basis for the minimum sample size estimation. Categorical data are presented as a number with percentage, whereas quantitative data is presented as median (range) or mean \pm standard error (SE) respectively. Mann-Whitney $U$ test or Student $t$ test was used for continuous variables analysis, whereas chi-square test or Fisher's exact test was used for categorical variables analysis as appropriate. Only those potential risk factors $(p<0.05)$ on univariate analysis were enclosed into the final multivariate logistic regression analysis. Binary multivariate stepwise logistic regression model was used in this study. The continuous data used in the logistic model was divided into two groups (high vs low, using the median value as the cutoff value). The predictive value of $\Delta \mathrm{Alb}$ for postoperative severe complications was evaluated by receiver operating characteristic (ROC) curve analysis.

\section{Results}

\section{Patient characteristics}

Of 218 consecutive patients, 25 patients were excluded according to the exclusion criteria (6 tumor metastases found intra-operatively, 7 emergency operations, 4 with neo-adjuvant treatment, 3 laparoscopic conversions to laparotomy, and 5 lack of albumin values within postoperative 2 days), which is shown in Fig. 1. A total of 193 patients were finally included in the multivariate analysis dataset, of which $60.1 \%(116 / 193)$ were male patients, as shown in Table 1. Eventually, 38 patients had postoperative severe complications according to the Clavien-Dindo grade, with an incidence of $19.7 \%$. Postoperative severe complications included 6 severe bleedings, 9 anastomotic leakages, 18 severe infections, 4 bowel obstructions, and 1 severe cardiopulmonary failure. The mean age and BMI of the total cohort were 53.4 years and $20.8 \mathrm{~kg} / \mathrm{m}^{2}$ respectively. Patients with severe complications had an older age 


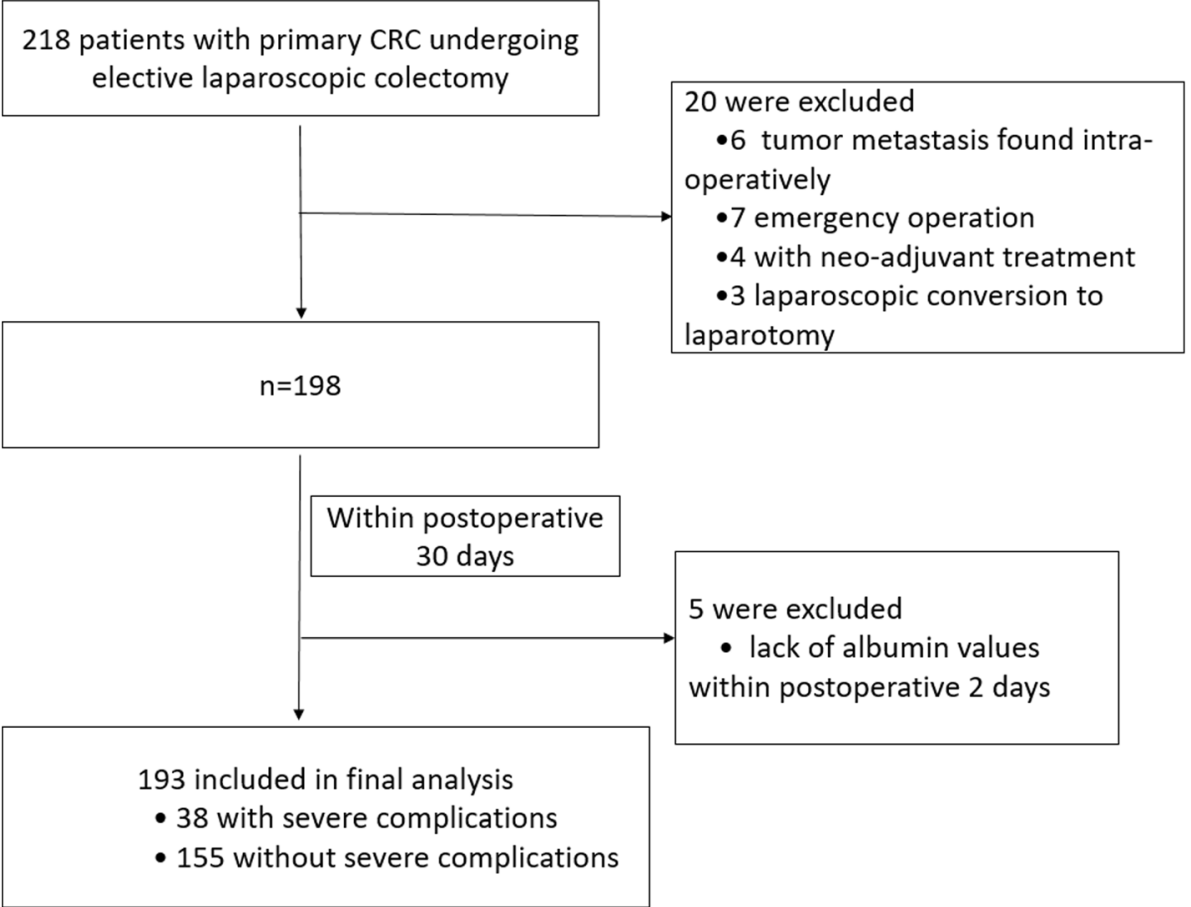

Fig. 1 Flow chart of the cases analyzed

than those without severe complications $(52.5 \pm 11.7$ vs $57.2 \pm 12.1, p=0.029)$. Patients with the comorbidity of hypertension were at an increased risk of postoperative severe complications $(p=0.029)$. The history of previous abdominal surgery was also significantly associated with increased severe complications $(p=0.030)$. In addition, patients with severe complications had a longer duration of operation $(p=0.043)$ and more estimated intraoperative blood loss $(p=0.032)$. Those patients with perioperative blood transfusion were also frequent in the patients with severe complications $(p=0.026)$. No significant differences were found in gender, BMI, smoking habits, ASA class, tumor location, AJCC stage, intraoperative fluid utilization, number of lymph nodes resection, and time to first flatus between the patients with or without severe complications (all $p>0.05$ ).

\section{Laboratory tests}

As summarized in Table 2, the patients with severe complications had a significantly higher $\Delta$ Alb value than those without severe complications $(13.3 \pm 2.9$ vs $19.0 \pm 3.5$, $p<0.001)$. In addition, higher $\Delta \mathrm{Hb}(p=0.034)$ and peak CRP level within POD3 $(p=0.005)$ were also significantly associated with postoperative severe complications.

\section{Risk factors associated with postoperative severe complications}

Subsequently, univariate and multivariate analyses were performed to investigate potential risk factors for postoperative severe complications. These nine potential risk factors mentioned above (Tables 1 and 2) were enclosed into the univariate analysis. Of the nine factors, five (duration of operation, perioperative blood transfusion, $\Delta \mathrm{Hb}, \Delta \mathrm{Alb}$, and peak $\mathrm{CRP}$ within POD3) were significantly associated with postoperative severe complications. In the final multivariate logistic regression analysis, $\Delta$ Alb was the only independent factor associated with postoperative severe complications (OR 1.66, 95\%CI 1.18-2.33, $p=0.003$, see Table 3).

\section{Predictive value of $\Delta \mathrm{Alb}$ for postoperative severe complications}

The receiver operator characteristic (ROC) curve analysis was applied to establish the predictive power of $\Delta \mathrm{Alb}$ for postoperative severe complications. As illustrated in Fig. 2, the area under the curve (AUC) of $\triangle \mathrm{Alb}$ was 0.916 , with the cutoff value of $17.3 \%$. The sensitivity and specificity were 0.842 and 0.858 , respectively $(p<0.001)$.

\section{Discussion}

In this current study, we focused on the potential association between serum Alb, an acute phase protein, and postoperative severe complications within POD 30. Our results revealed that a greater change in the serum Alb within POD 2 was an independent risk factor associated with postoperative severe complications. Postoperative severe complications were with a rate of $19.7 \%$ in this 
Table 1 Clinicopathological characteristics of CRC patients with severe complications or not

\begin{tabular}{|c|c|c|c|}
\hline \multirow[t]{2}{*}{ Parameters } & \multicolumn{2}{|c|}{ Postoperative severe complications } & \multirow[t]{2}{*}{$p$ value } \\
\hline & No $(n=155)$ & Yes $(n=38)$ & \\
\hline Age (year) & $52.5 \pm 11.7$ & $57.2 \pm 12.1$ & $0.029^{*}$ \\
\hline \multicolumn{4}{|l|}{ Gender, $n(\%)$} \\
\hline Male & $92(59.4)$ & $24(63.2)$ & \\
\hline Female & $63(40.6)$ & $14(36.8)$ & 0.67 \\
\hline $\mathrm{BMI}\left(\mathrm{kg} / \mathrm{m}^{2}\right)$ & $20.9 \pm 1.3$ & $20.6 \pm 1.5$ & 0.218 \\
\hline \multicolumn{4}{|l|}{ Comorbidities, $n$ (\%) } \\
\hline Hypertension & $28(18.1)$ & $13(34.2)$ & $0.029^{*}$ \\
\hline Diabetes mellitus & $18(11.6)$ & $6(15.8)$ & 0.48 \\
\hline \multicolumn{4}{|l|}{ Smoking status, $n(\%)$} \\
\hline Current smoker & $17(11.0)$ & $7(18.4)$ & \\
\hline History of smoking & $14(9.0)$ & $5(13.2)$ & \\
\hline Never & 124(80.0) & 26(68.4) & 0.30 \\
\hline \multicolumn{4}{|l|}{ ASA class, $n(\%)$} \\
\hline$\|$ & $121(78.1)$ & $28(73.7)$ & \\
\hline III & $34(21.9)$ & $10(26.3)$ & 0.56 \\
\hline Previous abdominal surgery, $n(\%)$ & $38(24.5)$ & $16(42.1)$ & $0.030^{*}$ \\
\hline \multicolumn{4}{|l|}{ Tumor location, $n(\%)$} \\
\hline Colon & $90(58.1)$ & $20(52.6)$ & \\
\hline Rectum & $65(41.9)$ & $18(47.4)$ & 0.54 \\
\hline \multicolumn{4}{|l|}{ AJCC stage, $n(\%)$} \\
\hline$|-| \mid$ & $88(56.8)$ & $18(47.4)$ & \\
\hline III & $67(43.2)$ & $20(52.6)$ & 0.30 \\
\hline Duration of operation (min) & $203.4 \pm 30.1$ & $215.4 \pm 41.2$ & $0.043^{*}$ \\
\hline Estimated blood loss (mL) & $180(60-710)$ & $240(80-850)$ & $0.032^{*}$ \\
\hline Intraoperative fluid utilization (mL) & $1800(1300-3100)$ & $1900(1400-2900)$ & 0.28 \\
\hline Number of lymph nodes resection & $10.7 \pm 5.2$ & $10.9 \pm 5.8$ & 0.836 \\
\hline Perioperative blood transfusion, $n(\%)$ & $34(21.9)$ & $15(39.5)$ & $0.026^{*}$ \\
\hline Time to first flatus (d) & $3.0 \pm 0.6$ & $3.1 \pm 0.9$ & 0.410 \\
\hline
\end{tabular}

present study, which was relatively similar to previous reports [13].

As reported by previous data, preoperative or early postoperative hypoalbuminemia is accepted as a risk factor for postoperative complications after a gastrointestinal operation, especially surgical site infections $[8,14]$. However, whether the relative change in perioperative serum Alb closely correlates with postoperative complications remains unclear. As widely proved, decreased Alb expression after the surgery is always observed due to the systemic inflammatory response syndrome [15]. As illustrated by a recent pilot study, postoperative albumin concentration is significantly decreased and it is suggested as a response biomarker for operation stress [16], which is quite in accordance with our results. In addition, perioperative hemodilution and fluid overload are also important explanations for decreased serum Alb after operation [17]. As summarized by previous studies, the decreased Alb level is ascribed to various factors, including Alb redistribution, perioperative blood loss, catabolism, and hemodilution [17]. A recent study has revealed increased postoperative CRP level as an independent factor associated with Alb reduction [7], which strongly suggests the close association between Alb and inflammatory response. Accumulating evidence has suggested CRP as a predictor for postoperative complications and prognosis after abdominal surgery [18]. Our univariate analysis showed 
Table 2 Laboratory tests in CRC patients with severe complications or not

\begin{tabular}{|c|c|c|c|}
\hline \multirow[t]{2}{*}{ Laboratory tests } & \multicolumn{2}{|c|}{ Postoperative severe complications } & \multirow[t]{2}{*}{$p$ value } \\
\hline & No $(n=155)$ & Yes $(n=38)$ & \\
\hline Preoperative $\mathrm{Hb}$ & $117.5 \pm 7.5$ & $116.4 \pm 8.4$ & 0.43 \\
\hline Preoperative Alb & $39.2 \pm 4.6$ & $37.8 \pm 5.1$ & 0.10 \\
\hline Preoperative Hct & $0.42 \pm 0.07$ & $0.43 \pm 0.05$ & 0.41 \\
\hline Preoperative CRP & $10.8 \pm 3.5$ & $11.1 \pm 2.7$ & 0.62 \\
\hline$\Delta \mathrm{Hb}(\%)$ & $14.1 \pm 4.2$ & $15.7 \pm 3.9$ & $0.034^{*}$ \\
\hline$\triangle$ Alb (\%) & $13.3 \pm 2.9$ & $19.0 \pm 3.5$ & $<0.001^{*}$ \\
\hline$\Delta \mathrm{Hct}(\%)$ & $-10.2 \pm 12.4$ & $-11.4 \pm 13.6$ & 0.60 \\
\hline Peak CRP within POD3 & $89.4 \pm 6.8$ & $104.4 \pm 9.1$ & $0.005^{*}$ \\
\hline
\end{tabular}

$C R C$ colorectal cancer, $\mathrm{Hb}$ hemoglobin, Alb albumin, $\mathrm{Hct}$ hematocrit, CRP Creactive protein, $P O D$ postoperative day. $p$ values were calculated by MannWhitney $U$ or $t$ test. ${ }^{*} p$ value $<0.05$

that peak CRP within POD3 was significantly associated with postoperative severe complications; however, the final multivariate results did not suggest its predictive role.

Previous reports have revealed that malnutrition and inflammatory response strongly correlate with severe postoperative complications [19]. As for those patients with malignancy, the development of inflammatory response is closely associated with decreased Alb and total lymphocyte count [20,21]. Serum Alb is reported to act various roles, including cell growth stabilization, DNA replication, sex hormone homeostasis maintaining, and systemic inflammation modulation[22].

A recent study in patients with cancer has shown that hypoalbuminemia reflects the condition of malnutrition and immunosuppression, and it is at an increased risk of

Table 3 Univariate and multivariate logistic regression analyses of perioperative factors on postoperative severe complications

\begin{tabular}{|c|c|c|c|c|}
\hline \multirow[t]{2}{*}{ Variables } & \multicolumn{2}{|l|}{ Univariate } & \multicolumn{2}{|l|}{ Multivariate } \\
\hline & OR (95\% Cl) & $p$ value & OR (95\% Cl) & $p$ value \\
\hline Age & $1.02(0.98-1.05)$ & 0.75 & & \\
\hline Hypertension & $1.22(0.76-1.94)$ & 0.43 & & \\
\hline $\begin{array}{l}\text { Previous abdominal } \\
\text { surgery }\end{array}$ & $1.40(0.93-2.04)$ & 0.12 & & \\
\hline $\begin{array}{l}\text { Duration of } \\
\text { operation }\end{array}$ & $1.38(1.03-1.94)$ & $0.043^{*}$ & $0.93(0.57-1.49)$ & 0.72 \\
\hline $\begin{array}{l}\text { Estimated blood } \\
\text { loss }\end{array}$ & $0.82(0.58-1.14)$ & 0.23 & & \\
\hline $\begin{array}{l}\text { Perioperative } \\
\text { blood transfusion }\end{array}$ & $2.46(1.47-4.02)$ & $0.012^{*}$ & $1.33(0.62-2.77)$ & 0.43 \\
\hline$\Delta \mathrm{Hb}$ & $2.34(1.29-4.61)$ & $0.009^{*}$ & $1.66(0.68-3.89)$ & 0.23 \\
\hline$\Delta$ Alb & $1.68(1.22-2.32)$ & $0.002^{*}$ & $1.66(1.18-2.33)$ & $0.003^{*}$ \\
\hline $\begin{array}{l}\text { Peak CRP within } \\
\text { POD3 }\end{array}$ & $0.70(0.48-0.99)$ & $0.032^{*}$ & $1.12(0.65-1.94)$ & 0.65 \\
\hline
\end{tabular}

$H b$ hemoglobin, $A l b$ albumin, CRP C-reactive protein, $P O D$ postoperative day, $O R$ odds ratio, $\mathrm{Cl}$ confidence interval. ${ }^{*} p$ value $<0.05$

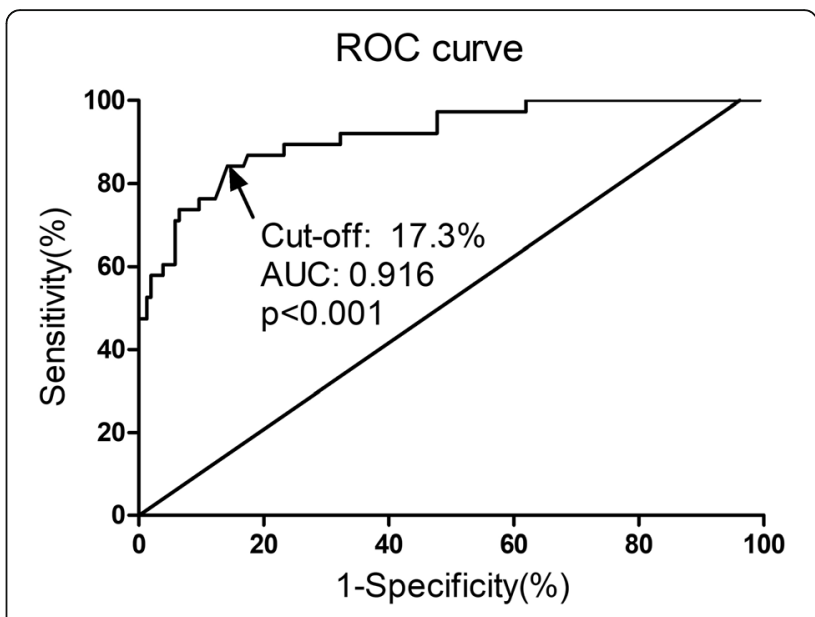

Fig. 2 The predictive value of $\triangle$ Alb for postoperative severe complications by ROC analysis. AUC 0.916 , cutoff value $17.3 \%$, sensitivity 0.842 , specificity $0.858, p<0.001$. Alb albumin, ROC receiver operating characteristics, $A \cup C$ area under the curve

disease severity, tumor progression, and poor prognosis [23]. Furthermore, serum Alb level has also been widely used in various prognostic indexes, including a prognostic nutritional index (PNI) [24], a systematic inflammation index (IPI) [25], and Naples prognostic score (NPS) [26]. Serum Alb plays important roles in colloid osmotic pressure maintenance, free radical scavenging, and capillary membrane permeability alteration [27]. The important physiologic functions of serum Alb may be potential explanations for the predictive role of $\Delta$ Alb for postoperative severe complications.

This study has some certain limitations. First, our data set came from a retrospective and single-center design, and the sample size was relatively small. Second, this study did not take the impacts of liver function and body fluid volume on serum albumin concentrations into consideration. Nevertheless, to our knowledge, this is the first study that highlighted the significance of $\Delta \mathrm{Alb}$ for postoperative severe complications. Of course, additional larger-scale prospective studies and basic researches are needed to confirm our results.

\section{Conclusions}

In conclusion, our results revealed that the $\Delta \mathrm{Alb}$ was an independent risk factor for severe complications in CRC patients after curative laparoscopic surgery. The surgeon and anesthetist could differentiate the patients according to the reduction trend of serum Alb and treat them accordingly. Predicting the risk of postoperative severe complications with serum Alb detections helps the surgeon in the outcome evaluation. The evaluation of the nutritional status prior to surgery is of great importance and if possible, correcting the deficit is recommended by our results. 


\section{Abbreviations}

AJCC: American Joint Committee on Cancer; Alb: Albumin; ASA: American Society of Anesthesiologists; BMI: Body mass index; Cl: Confidence interval; CRC: Colorectal cancer; CRP: C-reactive protein; Hb: Hemoglobin; Hct: Hematocrit; OR: Odds ratio; POD: Postoperative day; ROC: Receiver operating characteristic; SE: Standard error

\section{Funding}

This work was supported partly by funding from the National Natural Science Foundation of China (Grant no. 81600434), Jiangsu Natural Science Foundation (Grant no. BK20160572 and BK20170358), and Jiangsu Provincia Medical Youth Talent (Grant no. QNRC2016514).

\section{Availability of data and materials}

Please contact the author for data requests.

\section{Authors' contributions}

YW, HGW, and JGJ participated in the conception and design, data collection, statistical analysis, and writing of the manuscript. XFC and QHL participated in the conception and design and data collection. All authors read and approved the final manuscript.

\section{Ethics approval and consent to participate}

This study protocol was approved by the Medical Institutional Ethics Committee of Jiangsu province. All patients included were required to offer written informed consent.

\section{Consent for publication}

Not applicable.

\section{Competing interests}

The authors' declare that they have no competing interests.

\section{Publisher's Note}

Springer Nature remains neutral with regard to jurisdictional claims in published maps and institutional affiliations.

Received: 7 June 2018 Accepted: 10 September 2018

Published online: 25 September 2018

\section{References}

1. Global Burden of Disease Cancer C, Fitzmaurice C, Dicker D, Pain A, Hamavid H, Moradi-Lakeh M, et al. The global burden of cancer 2013. JAMA Oncol. 2015;1(4):505-27.

2. Papamichael D, Audisio RA, Glimelius B, de Gramont A, Glynne-Jones R, Haller D, et al. Treatment of colorectal cancer in older patients: International Society of Geriatric Oncology (SIOG) consensus recommendations 2013. Ann Oncol. 2015;26(3):463-76

3. Henneman D, Snijders HS, Fiocco M, van Leersum NJ, Kolfschoten NE, Wiggers T, et al. Hospital variation in failure to rescue after colorectal cancer surgery: results of the Dutch Surgical Colorectal Audit. Ann Surg Oncol. 2013:20(7):2117-23.

4. Ortega-Deballon P, Radais F, Facy O, d'Athis P, Masson D, Charles PE, et al. C-reactive protein is an early predictor of septic complications after elective colorectal surgery. World J Surg. 2010;34(4):808-14

5. Rettig TC, Verwijmeren L, Dijkstra IM, Boerma D, van de Garde EM, Noordzij PG. Postoperative interleukin-6 level and early detection of complications after elective major abdominal surgery. Ann Surg. 2016:263(6):1207-12.

6. Warschkow R, Beutner U, Steffen T, Muller SA, Schmied BM, Guller U, et al. Safe and early discharge after colorectal surgery due to C-reactive protein: a diagnostic meta-analysis of 1832 patients. Ann Surg. 2012;256(2):245-50.

7. Ge X, Dai X, Ding C, Tian H, Yang J, Gong J, et al. Early postoperative decrease of serum albumin predicts surgical outcome in patients undergoing colorectal resection. Dis Colon Rectum. 2017;60(3):326-34

8. Moghadamyeghaneh Z, Hwang G, Hanna MH, Phelan MJ, Carmichael JC, Mills SD, et al. Even modest hypoalbuminemia affects outcomes of colorectal surgery patients. Am J Surg. 2015:210(2):276-84.

9. Watanabe T, Itabashi M, Shimada Y, Tanaka S, Ito Y, Ajioka Y, et al. Japanese Society for Cancer of the Colon and Rectum (JSCCR) guidelines 2014 for treatment of colorectal cancer. Int J Clin Oncol. 2015;20(2):207-39.
10. Cao X, Zhao G, Yu T, An Q, Yang H, Xiao G. Preoperative prognostic nutritional index correlates with severe complications and poor survival in patients with colorectal cancer undergoing curative laparoscopic surgery: a retrospective study in a single Chinese institution. Nutr Cancer. 2017;69(3): 454-63.

11. Clavien PA, Barkun J, de Oliveira ML, Vauthey JN, Dindo D, Schulick RD, et al. The Clavien-Dindo classification of surgical complications: five-year experience. Ann Surg. 2009;250(2):187-96.

12. Spolverato G, Kim Y, Ejaz A, Frank SM, Pawlik TM. Effect of relative decrease in blood hemoglobin concentrations on postoperative morbidity in patients who undergo major gastrointestinal surgery. JAMA Surg. 2015;150(10):949-56

13. Boer BC, de Graaff F, Brusse-Keizer M, Bouman DE, Slump CH, Slee-Valentijn $\mathrm{M}$, et al. Skeletal muscle mass and quality as risk factors for postoperative outcome after open colon resection for cancer. Int J Color Dis. 2016:31(6): $1117-24$.

14. Lee Jl, Kwon M, Roh JL, Choi JW, Choi SH, Nam SY, et al. Postoperative hypoalbuminemia as a risk factor for surgical site infection after oral cancer surgery. Oral Dis. 2015;21(2):178-84

15. Fleck A, Raines G, Hawker F, Trotter J, Wallace PI, Ledingham IM, et al. Increased vascular permeability: a major cause of hypoalbuminaemia in disease and injury. Lancet. 1985;1(8432):781-4.

16. Hubner M, Mantziari S, Demartines N, Pralong F, Coti-Bertrand P, Schafer M. Postoperative albumin drop is a marker for surgical stress and a predictor for clinical outcome: a pilot study. Gastroenterol Res Pract. 2016;2016: 8743187.

17. Ryan AM, Hearty A, Prichard RS, Cunningham A, Rowley SP, Reynolds JV. Association of hypoalbuminemia on the first postoperative day and complications following esophagectomy. J Gastrointest Surg. 2007;11(10): 1355-60.

18. Watt DG, Horgan PG, McMillan DC. Routine clinical markers of the magnitude of the systemic inflammatory response after elective operation: a systematic review. Surgery. 2015:157(2):362-80.

19. Mohri Y, Inoue Y, Tanaka K, Hiro J, Uchida K, Kusunoki M. Prognostic nutritional index predicts postoperative outcome in colorectal cancer. World J Surg. 2013;37(11):2688-92.

20. Erdman SE, Poutahidis T. Roles for inflammation and regulatory T cells in colon cancer. Toxicol Pathol. 2010;38(1):76-87.

21. Crumley AB, Stuart RC, McKernan M, McMillan DC. Is hypoalbuminemia an independent prognostic factor in patients with gastric cancer? World J Surg 2010;34(10):2393-8.

22. Gupta D, Lis CG. Pretreatment serum albumin as a predictor of cancer survival: a systematic review of the epidemiological literature. Nutr $J$ 2010;9:69

23. Nazha B, Moussaly E, Zaarour M, Weerasinghe C, Azab B. Hypoalbuminemia in colorectal cancer prognosis: nutritional marker or inflammatory surrogate? World J Gastrointest Surg. 2015;7(12):370-7.

24. Tokunaga R, Sakamoto Y, Nakagawa S, Miyamoto Y, Yoshida N, Oki E, et al. Prognostic nutritional index predicts severe complications, recurrence, and poor prognosis in patients with colorectal cancer undergoing primary tumor resection. Dis Colon Rectum. 2015;58(11):1048-57.

25. Hong T, Shen D, Chen X, Cai D, Wu X, Hua D. A novel systematic inflammation related index is prognostic in curatively resected nonmetastatic colorectal cancer. Am J Surg. 2018;216(3):450-7.

26. Galizia G, Lieto E, Auricchio A, Cardella F, Mabilia A, Podzemny V, et al. Naples prognostic score, based on nutritional and inflammatory status, is an independent predictor of long-term outcome in patients undergoing surgery for colorectal cancer. Dis Colon Rectum. 2017;60(12):1273-84.

27. Margarson MP, Soni N. Serum albumin: touchstone or totem? Anaesthesia. 1998:53(8):789-803. 